\title{
DYNAMICAL HYSTERESIS WITHOUT STATIC HYSTERESIS: SCALING LAWS AND ASYMPTOTIC EXPANSIONS*
}

\author{
GUILLERMO H. GOLDSZTEIN ${ }^{\dagger}$, FERNANDO BRONER ${ }^{\dagger}$, AND STEVEN H. STROGATZ
}

\begin{abstract}
We study dynamical hysteresis in a simple class of nonlinear ordinary differential equations, namely first-order equations subject to sinusoidal forcing. The assumed nonlinearities are such that the area of the hysteresis loop vanishes as the forcing frequency tends to zero; in other words, there is no static hysteresis. Using regular and singular perturbation techniques, we derive the first term in the asymptotic expansion for the loop area as a function of the driving frequency, in the limit of both large and small frequency. Although the theory was originally motivated by experiments on bistable semiconductor lasers, it is applied here to explain (and in some cases, to correct) the scaling laws that were recently reported in numerical studies of mean-field kinetic Ising models of ferromagnets.
\end{abstract}

Key words. dynamical hysteresis, scaling laws, kinetic Ising model

AMS subject classifications. $34 \mathrm{C} 15,34 \mathrm{E} 05,82 \mathrm{C} 20$

PII. S0036139995290733

1. Introduction. Hysteresis is ubiquitous in mechanical, chemical, biological, electronic, optical, and magnetic systems. It is technologically useful for switches and memory devices and is also of fundamental scientific and mathematical interest.

Figure 1.1(a) illustrates a familiar form of hysteresis, in which a bistable system is driven adiabatically by an external periodic forcing. The dashed curve represents the branches of equilibria that would be obtained if the control parameter $\mathcal{E}$ were held fixed. If, instead, we vary $\mathcal{E}$ extremely slowly, say according to $\mathcal{E}=E \sin \Omega t$ with $\Omega \rightarrow 0$, the state variable $x$ essentially tracks the curve of equilibria, except when $\mathcal{E}$ crosses a turning point and the system jumps from one stable branch to the other. The resulting closed curve in the $(\mathcal{E}, x)$ plane is called a hysteresis loop. Since this hysteresis loop has nonzero area even in the limit of zero-frequency driving, we say that this system exhibits static hysteresis.

The situation becomes more interesting if we allow the switching parameter $\mathcal{E}$ to vary slowly, but not quite adiabatically, with some frequency $\Omega>0$. Then, as shown in Figure 1.1(b), the hysteresis loop acquires extra area because the system is unable to relax completely. This extra area is known as the dynamical hysteresis area. In many applications it is a physically important quantity. For instance, in magnetic and optical switches, the dynamical hysteresis area provides a measure of the additional power dissipated by repetitive switching at a frequency $\Omega$. For this reason, and for reasons of intrinsic mathematical interest, one would like to know how this area depends on $\Omega$.

In the last few years there have been many experimental $[8,9,11,12]$ and theoretical $[1-7,9-11,13-24]$ investigations of dynamical hysteresis, often in the context of magnetic spin systems driven by an oscillating external field. Such spin systems

* Received by the editors August 23, 1995; accepted for publication (in revised form) March 18, 1996.

http://www.siam.org/journals/siap/57-4/29073.html

${ }^{\dagger}$ Department of Mathematics, Massachusetts Institute of Technology, Cambridge, MA 02139.

¥Department of Theoretical and Applied Mechanics and Center for Applied Mathematics, Kimball Hall, Cornell University, Ithaca, NY 14853 (strogatz@cornell.edu). The research of this author was supported in part by National Science Foundation Presidential Young Investigator Award DMS9057433 and grant DMS-9500948. 

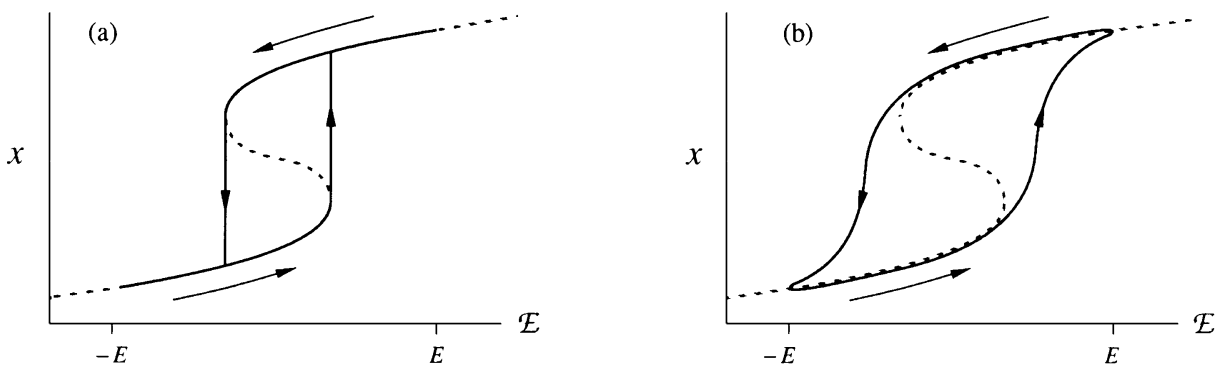

FIG. 1.1. (a) Static hysteresis loop (solid curve) for $\mathcal{E}=E \sin \Omega t$, with $\Omega \rightarrow 0$. Dashed curve, equilibria for fixed values of $\mathcal{E}$. (b) Dynamical hysteresis loop for $\Omega>0$.

are formidable to analyze because they involve many coupled degrees of freedom, as well as stochastic effects due to thermal fluctuations. As an alternative strategy, some authors have investigated dynamical hysteresis in the simplest possible setting of deterministic systems with only one state variable. These systems pose interesting mathematical challenges in their own right, and they have been shown to provide useful insight into the dynamics of bistable semiconductor lasers and mean-field models of magnetic systems.

For instance, the dynamical hysteresis area was calculated analytically by Jung, Gray, Roy, and Mandel [11] for the model equation

$$
\dot{x}=\lambda x-b x^{3}+E \sin \Omega t,
$$

where $\lambda, b, E$, and $\Omega$ are all positive constants, and $E$ is large enough that the system is repeatedly carried past the turning points, as in Figure 1.1(b). Jung, Gray, Roy, and Mandel [11] found that in the limit $\Omega \rightarrow 0$, the area $A(\Omega)$ of the hysteresis loop $(E \sin \Omega t, x(t))$ obeys the scaling law

$$
A(\Omega)-A(0) \propto \Omega^{\frac{2}{3}},
$$

where $A(0)$ is the area of the static hysteresis loop. They also showed that this scaling law provides a good fit to their experimental measurements on a bistable semiconductor laser system.

A natural extension of this research involves the effects of bifurcations on the scaling laws. Figure 1.2 illustrates one common scenario, often associated with phase transitions. Suppose that in addition to the switching parameter $\mathcal{E}$ there is also a bifurcation parameter, analogous to the bias current in a bistable laser [9] or the temperature in a magnetic spin system [14]. As this bifurcation parameter varies, the curve of steady states $x$ versus $\mathcal{E}$ changes from triple valued to single valued. At a threshold value of the parameter, the curve develops a vertical inflection point (Figure 1.2(b)). The single-valued dashed curves of Figures 1.2(b) and 1.2(c) cannot exhibit static hysteresis, but they can still exhibit dynamical hysteresis, with loops of the shape indicated in Figure 1.2. Several questions arise: in the absence of static hysteresis, are there still scaling laws for the dynamical hysteresis area and, if so, what are the new exponents?

In this paper we answer these questions analytically for equations of the form

$$
\dot{x}=-F(x)+E \sin \Omega t,
$$



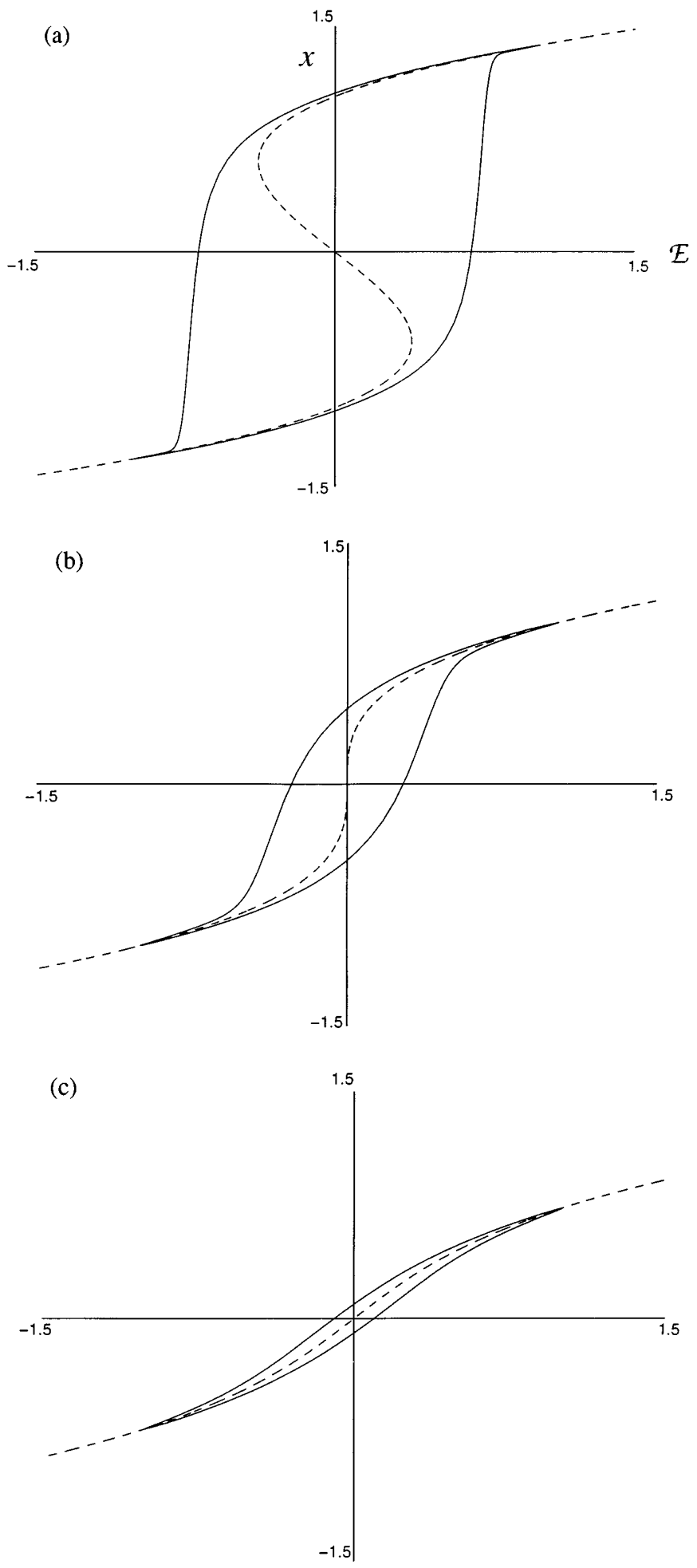

FIG. 1.2. Dynamical hysteresis loops (solid curves) for the model system (1.3) with $E=1, \Omega=$ 0.1 , and $F(x)=-\lambda x+x^{3}$. Here $\lambda$ is the bifurcation parameter. Dashed curve, equilibria for fixed values of $\mathcal{E}$. (a) $\lambda=1$. (b) Threshold case: $\lambda=0$. (c) $\lambda=-1$. 
where $F(x)$ is a continuously differentiable function that is strictly increasing (so there is no static hysteresis). In section 2 we prove that (1.3) has a globally attracting periodic solution for any $\Omega>0$, and we calculate the area of the hysteresis loop for the special case where $F(x)$ is linear. This solvable case provides a benchmark for later results.

In sections 3 and 4 we analyze (1.3) in the limit of small $\Omega$. As the discussion above suggests, there is a crucial distinction between the "generic" case when $F^{\prime}(x)>0$ for all $x$ of interest (Figure 1.2(c)) and the "threshold" case when $F^{\prime}(x)=0$ for some $x$ (Figure 1.2(b)).

The generic case is analyzed in section 3, and the results are summarized in Proposition 3.1. We use regular perturbation theory to prove that the scaling law is

$$
A(\Omega) \propto \Omega
$$

as $\Omega \rightarrow 0$. Recently, in a collaboration with Hohl, van der Linden, and Roy [9], we found good agreement between this prediction and the scaling measured experimentally in a bistable semiconductor laser system.

The threshold case (section 4) is more delicate and requires singular perturbation theory. As shown in Proposition 4.1, the exponent in the scaling law depends on the order of the zero of $F^{\prime}(x)$. Our techniques yield the first term in an asymptotic expansion for $A(\Omega)$ itself, so we obtain the numerical prefactor in the power law as well as the exponent. Specifically, for systems of the form

$$
\dot{x}=-x|x|^{a}+\sin \Omega t,
$$

we prove that as $\Omega \rightarrow 0$

$$
A(\Omega) \sim \begin{cases}C_{1} \Omega & \text { if } 0<a<1, \\ -\frac{2}{3} \Omega \ln \Omega & \text { if } a=1, \\ C_{2} \Omega^{\frac{a+2}{2 a+1}} & \text { if } a>1 .\end{cases}
$$

The constants $C_{1}$ and $C_{2}$ are explicit definite integrals that depend on $a$ but not on $\Omega$. These asymptotic results are shown to agree with numerical calculations of $A(\Omega)$. Section 5 deals with (1.3) in the limit of large $\Omega$. We find that

$$
A(\Omega) \sim \frac{\pi E^{2}}{\Omega}
$$

as $\Omega \rightarrow \infty$. In this case it does not matter whether $F(x)$ has a zero derivative somewhere. In section 6 we indicate how to extend our results to more general systems, including higher-dimensional systems. Finally, in section 7 we apply the theory to explain (and in some cases, correct) the numerical observations of Luse and Zangwill [14] on mean-field kinetic Ising models of magnetic spin systems.

2. Preliminaries. We consider differential equations of the form

$$
\dot{x}=-F(x)+E \sin \Omega t,
$$

where $E$ and $\Omega$ are positive constants and $F(x)$ is a strictly increasing, continuously differentiable function that contains the interval $[-E, E]$ in its image.

Physically, (2.1) can be interpreted as the equation of motion for a heavily overdamped particle subject to a restoring force $-F(x)$ and a periodic drive of strength 
$E$ and frequency $\Omega$. One expects intuitively that after transients decay, such a system will always settle down to a forced oscillation of period $2 \pi / \Omega$. This is the content of the following lemma.

LEMMA 2.1. For any $\Omega$ there is a $2 \pi / \Omega$-periodic solution $z(t)$ to which all other solutions are attracted.

Proof. First we show that such a periodic solution exists, by finding a fixed point of the Poincaré map. Let $X\left(x_{0}, t\right)$ denote the solution of $(2.1)$ that satisfies $X\left(x_{0}, 0\right)=x_{0}$ and let $P\left(x_{0}\right)=X\left(x_{0}, 2 \pi / \Omega\right)$ denote the Poincaré map. Then $P$ is continuous since $X$ is a continuous function of $x_{0}$ and $t$. To show that $P$ has a fixed point, it suffices to show that $P$ maps some closed interval into itself. Specifically, let $x_{L}$ and $x_{U}$ be numbers such that $F\left(x_{L}\right)=-E$ and $F\left(x_{U}\right)=E$. If $x(t)$ is a solution of (2.1), $x(t)$ is increasing whenever $x(t)<x_{L}$ and decreasing whenever $x(t)>x_{U}$. Therefore $P\left(x_{L}\right) \geq x_{L}$ and $P\left(x_{U}\right) \leq x_{U}$, and so by continuity there exists a fixed point $x^{*}=P\left(x^{*}\right)$ in the interval $\left[x_{L}, x_{U}\right]$. Hence $z(t)=X\left(x^{*}, t\right)$ is a $2 \pi / \Omega$-periodic solution.

To show that all other solutions of (2.1) are attracted to $z(t)$, let $y(t)$ be another solution. Then

$$
\dot{z}-\dot{y}=-[F(z)-F(y)] .
$$

Since $F(x)$ is strictly increasing, $\dot{z}-\dot{y}<0$ if $z-y>0$ and $\dot{z}-\dot{y}>0$ if $z-y<0$, implying that $y(t)$ approaches $z(t)$ monotonically.

Throughout this paper, we will be concerned with the area of the dynamical hysteresis loop for the system (2.1). This area is defined as the area enclosed by the planar curve $(E \sin \Omega t, z(t))$ and is given by

$$
A(\Omega)=\left|\int_{0}^{\frac{2 \pi}{\Omega}} E \Omega \cos \Omega t z(t) d t\right| .
$$

It is instructive to begin with the special case where $F(x)$ is linear. The general solution of (2.1) for $F(x)=c x$ is given by

$$
x(t)=\frac{E}{\Omega^{2}+c^{2}}[c \sin \Omega t-\Omega \cos \Omega t]+B e^{-c t},
$$

where $B$ depends on the initial conditions. For $c>0$, all solutions are attracted to the $2 \pi / \Omega$-periodic solution

$$
z(t)=\frac{E}{\Omega^{2}+c^{2}}[c \sin \Omega t-\Omega \cos \Omega t] .
$$

In this case, the area of the dynamical hysteresis loop can be found explicitly for all $\Omega$. The result is

$$
A(\Omega)=\frac{E^{2} \pi \Omega}{c^{2}+\Omega^{2}} .
$$

Thus $A(\Omega) \propto \Omega$ as $\Omega \rightarrow 0$ and $A(\Omega) \propto \Omega^{-1}$ as $\Omega \rightarrow \infty$.

Figure 2.1 plots $A(\Omega)$ for $F(x)=x$ with $E=1$. For comparison, and to hint at what to expect when $F(x)$ is nonlinear, we have also plotted $A(\Omega)$ for $F(x)=$ $x^{3}$, as obtained by numerical integration. Note that the low-frequency behavior is qualitatively different - the graph starts with a vertical slope for cubic $F$ - but the high-frequency behavior is essentially the same. The goal of the following sections is to explain these asymptotic results for a broad class of functions $F(x)$. 


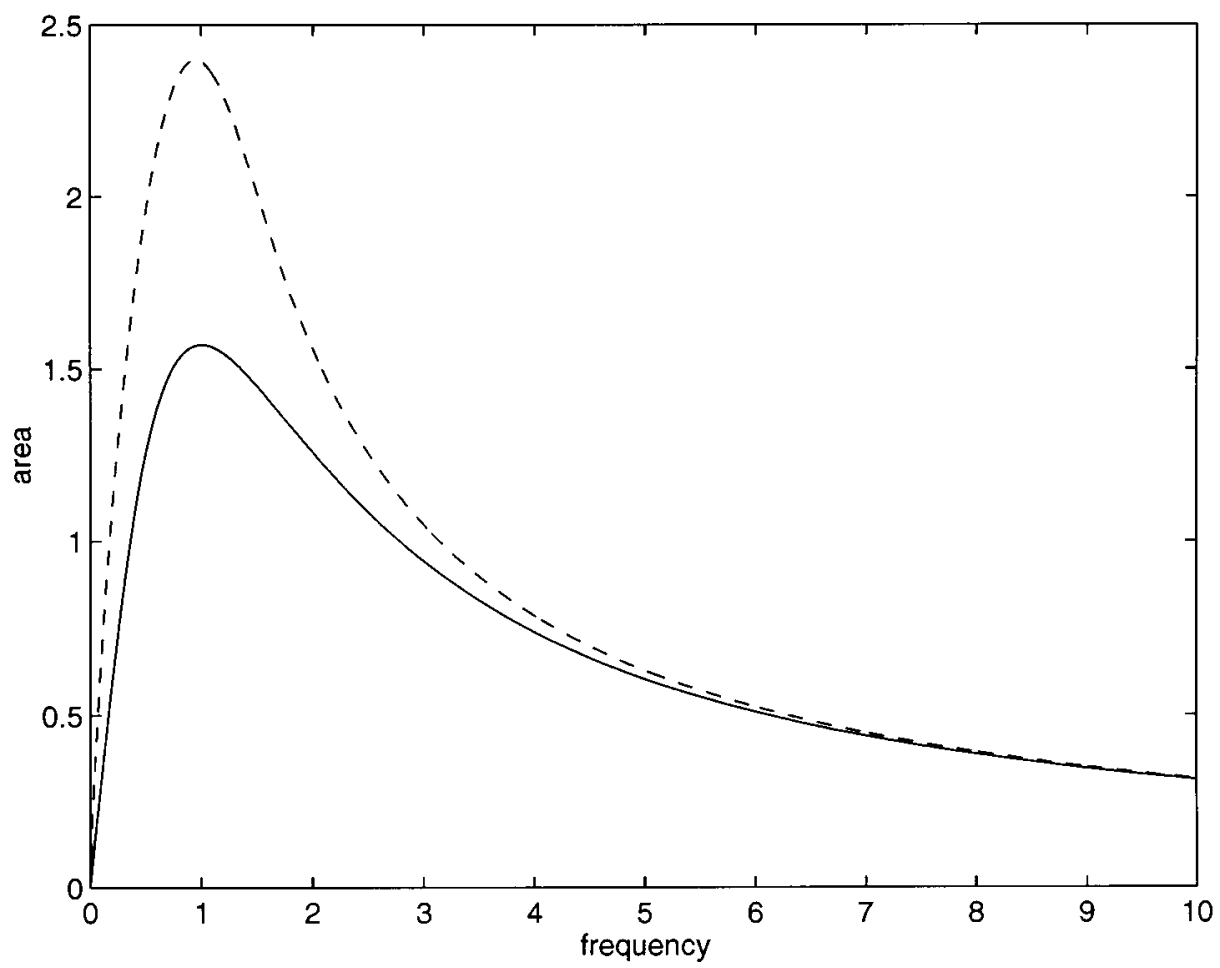

FIG. 2.1. Dynamical hysteresis area $A(\Omega)$ versus frequency $\Omega$ for $(2.1)$ with $E=1$. Solid line, $F(x)=x$; dashed line, $F(x)=x^{3}$.

3. Small $\Omega$ : Generic case. In this section we study the low-frequency behavior of $A(\Omega)$ for system (2.1), in the case where $F^{\prime}(x)$ is strictly positive. This is the generic case, given our assumption that the system does not have static hysteresis.

The strategy is to consider driving the system at a frequency $\Omega \ll \Omega_{0}$, where $\Omega_{0}$ is the slowest intrinsic relaxation rate at any point in the hysteresis loop. For such slow forcing, it suffices to use a quasi-static approximation for the limit cycle.

Proposition 3.1. Consider the system (2.1) and let

$$
\Omega_{0} \equiv \min _{-E \leq F(x) \leq E} F^{\prime}(x) .
$$

Suppose that $\Omega_{0}>0$. Then for $\Omega \ll \Omega_{0}$ the area of the dynamical hysteresis loop is proportional to $\Omega$.

Proof. Introduce the scaled variables $y=\Omega_{0} x / E$ and $\theta=\Omega t$. Then the system becomes

$$
\frac{d y}{d \theta}=\frac{-G(x)+\sin \theta}{\epsilon},
$$

where $\epsilon=\Omega / \Omega_{0}$ and $G(y)=F\left(\left(E / \Omega_{0}\right) y\right) / E$. The hypotheses imply that $\epsilon \ll 1$ and

$$
\min _{-1 \leq G(y) \leq 1} G^{\prime}(y)=1 .
$$

Let $u(\theta)$ be the periodic solution of (3.2) and expand $u$ as

$$
u=u_{0}+\epsilon u_{1}+o(\epsilon) .
$$


Substituting (3.4) in (3.2) and collecting powers of $\epsilon$, we obtain, at $O\left(\epsilon^{-1}\right)$

$$
0=-G\left(u_{0}(\theta)\right)+\sin \theta .
$$

At $O(1)$

$$
\frac{d u_{0}}{d \theta}=-G^{\prime}\left(u_{0}\right) u_{1},
$$

where we have used the fact that $G$ is differentiable. Solving for $u_{1}$ using (3.5) and (3.6) yields

$$
u_{1}=\frac{-\cos \theta}{\left(G^{\prime}\left(u_{0}\right)\right)^{2}} .
$$

From (3.3), (3.6), and (3.7) we see that $\left|u_{1}\right| \leq 1$ and $\left|d u_{0} / d \theta\right| \leq 1$, which shows that the approximations made are valid.

To complete the proof we now calculate the area of the dynamical hysteresis loop. The periodic solution of $(2.1)$ is $z=\left(E / \Omega_{0}\right) u$ and so

$$
\begin{aligned}
A(\Omega) & =\left|\int_{0}^{2 \pi} E \cos \theta z(\theta) d \theta\right| \\
& =\frac{E^{2}}{\Omega_{0}}\left|\int_{0}^{2 \pi} \cos \theta u(\theta) d \theta\right| \\
& \approx \Omega\left(\frac{E}{\Omega_{0}}\right)^{2} \int_{0}^{2 \pi}\left[\frac{\cos \theta}{G^{\prime}\left(u_{0}(\theta)\right)}\right]^{2} d \theta \\
& =\Omega E^{2} \int_{0}^{2 \pi}\left[\frac{\cos \theta}{F^{\prime}\left(x_{0}(\theta)\right)}\right]^{2} d \theta \\
& =\Omega \int_{0}^{2 \pi}\left(\frac{d x_{0}}{d \theta}\right)^{2} d \theta,
\end{aligned}
$$

where $x_{0}$ is defined by

$$
0=-F\left(x_{0}(\theta)\right)+E \sin \theta .
$$

In going from the second to the third line of (3.8), we have used the fact that $u_{0}$ depends on $\theta$ only through $\sin \theta$ (see (3.5)) to infer that $\int_{0}^{2 \pi} \cos \theta u_{0}(\theta) d \theta=0$. Since the integral in (3.8) is independent of $\Omega$, the proposition is established.

As a check, it is immediate to verify (3.8) when $F(x)=c x$ (the exact formula for this case is $(2.6))$.

Tables 3.1 and 3.2 show the results of numerical calculations with $E=1$ for two different choices of $F(x)$. The quantity $A\left(\Omega_{i}\right)$ is the value of the area obtained by direct numerical integration of the full system, whereas $A_{0}\left(\Omega_{i}\right)$ is the value predicted by the approximation (3.8). The integral in (3.8) must typically be evaluated numerically, but fortunately not much labor is required: only one numerical quadrature is required for each function $F$.

To test the predicted scaling more quantitatively, we introduce the quantity

$$
\beta_{i}=\frac{\ln \left(A\left(\Omega_{i}\right) / A\left(\Omega_{i+1}\right)\right)}{\ln \left(\Omega_{i} / \Omega_{i+1}\right)},
$$


TABLE 3.1

\begin{tabular}{|c|c|c|c|c|}
\hline \multicolumn{5}{|c|}{$F(x)=x$} \\
\hline$i$ & $\Omega_{i}$ & $A\left(\Omega_{i}\right)$ & $\beta_{i}$ & $A_{0}\left(\Omega_{i}\right)$ \\
\hline 1 & 0.1 & 0.3110 & 0.9892 & 0.3142 \\
2 & $0.1 \times 2^{-1}$ & 0.1567 & 0.9973 & 0.1571 \\
3 & $0.1 \times 2^{-2}$ & 0.0785 & 0.9993 & 0.0785 \\
4 & $0.1 \times 2^{-3}$ & 0.0393 & 0.9998 & 0.0393 \\
5 & $0.1 \times 2^{-4}$ & 0.0196 & 1.0000 & 0.0196 \\
6 & $0.1 \times 2^{-5}$ & 0.0098 & & 0.0098 \\
\hline
\end{tabular}

TABLE 3.2

\begin{tabular}{|c|l|l|l|l|}
\hline \multicolumn{5}{|c|}{$F(x)=x^{3}+x$} \\
\hline$i$ & $\Omega_{i}$ & $A\left(\Omega_{i}\right)$ & $\beta_{i}$ & $A_{0}\left(\Omega_{i}\right)$ \\
\hline 1 & 0.1 & 0.1757 & 0.9907 & 0.1773 \\
2 & $0.1 \times 2^{-1}$ & 0.0884 & 0.9975 & 0.0886 \\
3 & $0.1 \times 2^{-2}$ & 0.0443 & 0.9994 & 0.0443 \\
4 & $0.1 \times 2^{-3}$ & 0.0222 & 0.9999 & 0.0222 \\
5 & $0.1 \times 2^{-4}$ & 0.0111 & 1.0000 & 0.0111 \\
6 & $0.1 \times 2^{-5}$ & 0.0055 & & 0.0055 \\
\hline
\end{tabular}

which approximates the exponent in the scaling law $A(\Omega) \sim \Omega^{\beta}$. The tables show that for both choices of $F(x)$, the exponent $\beta \rightarrow 1$ as $\Omega \rightarrow 0$, as predicted.

Remark 1. We can also allow $F^{\prime}(x)$ take the value $\infty$ in Proposition 3.1. For example if $F(x)=x^{\frac{1}{3}}, A(\Omega)$ will still be proportional to $\Omega$.

4. Small $\Omega$ : Threshold case. In section 3 , we assumed that $\Omega_{0}>0$, which is equivalent to assuming that the system always relaxes exponentially fast, in the absence of forcing. However, this condition is violated at bifurcations of the type shown in Figure 1.2. In physical systems, this bifurcation arises in connection with secondorder phase transitions, where the resulting subexponential relaxation is known as "critical slowing down." We now investigate this threshold case by studying a model equation that captures the essential phenomena.

\subsection{Asymptotic formulas.}

PROPOSITION 4.1. Consider the system

$$
\dot{x}=-x|x|^{a}+\sin \Omega t .
$$

For $\Omega \ll 1$, the area of the dynamical hysteresis loop is given by the following asymptotic formulas:

$$
A(\Omega) \sim \begin{cases}C_{1} \Omega & \text { if } 0<a<1, \\ -\frac{2}{3} \Omega \ln \Omega & \text { if } a=1, \\ C_{2} \Omega^{\frac{a+2}{2 a+1}} & \text { if } a>1 .\end{cases}
$$

The prefactors $C_{1}$ and $C_{2}$ are given explicitly by definite integrals that depend on a but not on $\Omega$.

Proof. Make the change of variables $\theta=\Omega t$. System (4.1) becomes

$$
\frac{d x}{d \theta}=\frac{-x|x|^{a}+\sin \theta}{\Omega} .
$$

The lemma in section 2 implies that (4.3) has a globally attracting periodic solution $z(\theta)$. Naively imitating the approach used earlier to prove Proposition 3.1, we seek a 
perturbation expansion of $z(\theta)$ in powers of $\Omega$ :

$$
z=z_{0}+\Omega z_{1}+\Omega^{2} z_{2}+\cdots .
$$

Substituting this expansion in (4.3) and equating like powers of $\Omega$, we find

$$
z_{0}(\theta)=\sin \theta|\sin \theta|^{-\frac{a}{a+1}}
$$

and

$$
z_{1}(\theta)=\frac{-\cos \theta}{(a+1)^{2}|\sin \theta|^{\frac{2 a}{a+1}}} .
$$

The form of $z_{1}(\theta)$ exposes a difficulty that did not arise in the previous calculation: the series expansion is not uniformly valid, due to the $|\sin \theta|$ term in the denominator of $z_{1}$. Near $\theta=0$ and $\theta=\pi$, the expansion breaks down and boundary layers must be inserted. Therefore the series expansion (4.4) should be regarded as an outer expansion; it needs to be supplemented by inner expansions that are valid in the boundary layers, and then the inner and outer solutions must be joined by a matching procedure.

To find the appropriate scaling in the boundary layer near $\theta=0$, we observe that when $|\theta|=O\left(\Omega^{\frac{a+1}{2 a+1}}\right)$, all the terms $\Omega^{i} z_{i}$ in (4.4) become of the same order. This motivates the change of independent variables

$$
\phi=\frac{\theta}{\Omega^{\frac{a+1}{2 a+1}}} .
$$

Next we determine the appropriate scale for the dependent variable. For $\theta$ small but not too small, so that we are still in the outer regime where (4.4) is valid, we have

$$
\begin{aligned}
z & \approx \theta|\theta|^{-\frac{a}{a+1}}-\frac{\Omega|\theta|^{-\frac{2 a}{a+1}}}{(a+1)^{2}} \\
& =\Omega^{\frac{1}{2 a+1}}\left(\phi|\phi|^{-\frac{a}{a+1}}-\frac{|\phi|^{-\frac{2 a}{a+1}}}{(a+1)^{2}}\right),
\end{aligned}
$$

which suggests the change of dependent variables

$$
y=\frac{x}{\Omega^{\frac{1}{2 a+1}}}
$$

in the layer near $\theta=0$.

Then from (4.8) and (4.3), we have that for $|\theta| \ll 1$,

$$
z(\theta)=\Omega^{\frac{1}{2 a+1}} y_{1}\left(\frac{\theta}{\Omega^{\frac{a+1}{a+1}}}\right),
$$

where $y_{1}(\phi)$ satisfies

$$
\frac{d y_{1}}{d \phi}=-y_{1}\left|y_{1}\right|^{a}+\phi
$$

with asymptotic behavior

$$
y_{1} \approx \phi|\phi|^{-\frac{a}{a+1}}-\frac{|\phi|^{-\frac{2 a}{a+1}}}{(a+1)^{2}}
$$


as $\phi \rightarrow \pm \infty$. Here the asymptotic behavior is determined by the requirement to match the solution onto (4.8).

Using the same arguments but now for $\theta$ near $\pi$, we get for $|\theta-\pi| \ll 1$,

$$
z(\theta)=-\Omega^{\frac{1}{2 a+1}} y_{2}\left(\frac{\theta-\pi}{\Omega^{\frac{a+1}{2 a+1}}}\right) .
$$

Here $\phi=(\theta-\pi) / \Omega^{\frac{a+1}{2 a+1}}$ and $y_{2}(\phi)$ satisfies

$$
\frac{d y_{2}}{d \phi}=-y_{2}\left|y_{2}\right|^{a}+\phi
$$

with asymptotic behavior

$$
y_{2} \approx \phi|\phi|^{-\frac{a}{a+1}}-\frac{|\phi|^{-\frac{2 a}{a+1}}}{(a+1)^{2}}
$$

as $\phi \rightarrow \pm \infty$. This implies that $y_{1}(\phi)=y_{2}(\phi)$ since $\left|y_{1}(\phi)-y_{2}(\phi)\right|$ is decreasing (as seen by subtracting (4.14) from (4.11)) and $y_{1}(\phi)-y_{2}(\phi) \rightarrow 0$ as $\phi \rightarrow-\infty$.

Now we are in a position to calculate the area of the dynamical hysteresis loop:

$$
\begin{aligned}
A(\Omega) & =\left|\int_{0}^{2 \pi} \cos \theta z(\theta) d \theta\right| \\
& =\left|I_{\text {outer }}+I_{\text {inner }}\right|,
\end{aligned}
$$

where

$$
I_{\text {outer }}=\int_{\theta_{0}}^{\pi-\theta_{0}} \cos \theta z(\theta) d \theta+\int_{-\pi+\theta_{0}}^{-\theta_{0}} \cos \theta z(\theta) d \theta
$$

and

$$
I_{\text {inner }}=\int_{-\theta_{0}}^{\theta_{0}} \cos \theta z(\theta) d \theta+\int_{\pi-\theta_{0}}^{\pi+\theta_{0}} \cos \theta z(\theta) d \theta
$$

with

$$
\theta_{0}=\Omega^{\frac{a+1}{2 a+1}} \phi_{0}
$$

where $\phi_{0} \gg 1$ and $\theta_{0} \ll 1$ so that we can use the formula (4.4) to calculate $I_{\text {outer }}$ and (4.10) and (4.13) to calculate $I_{\text {inner }}$.

After some manipulations we obtain that

$$
\begin{aligned}
I_{\text {outer }} & \approx \frac{4 \Omega}{1-a^{2}}\left(\left(\sin \theta_{0}\right)^{\frac{1-a}{a+1}} \cos \theta_{0}-\int_{\theta_{0}}^{\frac{\pi}{2}}(\sin \theta)^{\frac{2}{a+1}} d \theta\right) \\
& \approx \frac{4 \Omega}{1-a^{2}}\left(\theta_{0}^{\frac{1-a}{a+1}}-\int_{0}^{\frac{\pi}{2}}(\sin \theta)^{\frac{2}{a+1}} d \theta\right) \\
& =\frac{4 \Omega}{1-a^{2}}\left(\phi_{0}^{\frac{1-a}{a+1}} \Omega^{\frac{1-a}{2 a+1}}-\int_{0}^{\frac{\pi}{2}}(\sin \theta)^{\frac{2}{a+1}} d \theta\right)
\end{aligned}
$$


and

$$
\begin{aligned}
I_{\text {inner }} & \approx \Omega^{\frac{a+2}{2 a+1}} \int_{-\phi_{0}}^{\phi_{0}} \cos \left(\Omega^{\frac{a+1}{2 a+1}} \phi\right)\left(y_{1}(\phi)+y_{2}(\phi)\right) d \phi \\
& \approx 2 \Omega^{\frac{a+2}{2 a+1}} \int_{-\phi_{0}}^{\phi_{0}} y_{1}(\phi) d \phi .
\end{aligned}
$$

The relative sizes of $I_{\text {inner }}$ and $I_{\text {outer }}$ depend on $a$. Let us begin with the case $a>1$. From (4.20), (4.21), and (4.22), we get

$$
I_{\text {outer }} \approx \frac{4 \phi_{0}^{\frac{1-a}{a+1}}}{1-a^{2}} \Omega^{\frac{a+2}{2 a+1}}
$$

and

$$
I_{\text {inner }} \approx \Omega^{\frac{a+2}{2 a+1}} I_{0}
$$

where we have introduced the integral

$$
I_{0}=2 \lim _{\phi_{0} \rightarrow \infty} \int_{-\phi_{0}}^{\phi_{0}} y_{1}(\phi) d \phi
$$

as an approximation to $2 \int_{-\phi_{0}}^{\phi_{0}} y_{1}(\phi) d \phi$, since $\phi_{0} \gg 1$.

We claim that $I_{0}$ exists and that it is finite and strictly negative. To prove these statements, we need to extract some properties of the unknown function $y_{1}(\phi)$. Recall that this function is defined as the solution of (4.11) with asymptotic behavior (4.12) as $\phi \rightarrow \pm \infty$. Let

$$
f(\phi)=\phi|\phi|^{-\frac{a}{a+1}}
$$

denote the leading term in the asymptotic expansion (4.12). Since $f$ is odd,

$$
\int_{-\phi_{0}}^{\phi_{0}} f(\phi) d \phi=0
$$

From (4.12) we see that $y_{1}-f(\phi)$ is integrable since $a>1$. Therefore $I_{0}$ exists and is finite because of (4.27). Next, to show that $I_{0}$ is strictly negative, we define $w(\phi)=y_{1}(\phi)-f(\phi)$ and observe that $I_{0}=2 \int_{-\infty}^{\infty} w(\phi) d \phi$. Thus it suffices to show that $w(\phi)<0$ for all $\phi$. We know that $w(\phi)<0$ for $\phi \rightarrow-\infty$ (see (4.12)), so the graph of $w(\phi)$ either remains below the line $w=0$ for all $\phi$, in which case we are done, or else the graph of $w(\phi)$ crosses up through $w=0$ with a nonnegative slope at some point $\phi_{1}$. But that leads to a contradiction, since $w^{\prime}\left(\phi_{1}\right)<0$ at any zero crossing. To see this, note that

$$
\frac{d w}{d \phi}=\frac{d y_{1}}{d \phi}-\frac{|\phi|^{-\frac{a}{a+1}}}{a+1} .
$$

From the definition of $f(\phi)$ and (4.11), $d y_{1} / d \phi=0$ at $\phi=\phi_{1}$, which implies that $w^{\prime}\left(\phi_{1}\right)<0$. Hence $w(\phi)<0$ for all $\phi$ and $I_{0}<0$, as claimed.

Now, since $a>1$ and $\phi_{0} \gg 1$, we have $\left|I_{\text {outer }}\right| \ll\left|I_{\text {inner }}\right|$ and hence

$$
A(\Omega) \approx \Omega^{\frac{a+2}{2 a+1}}\left|I_{0}\right| .
$$


At the end of this section, we will present a few results about the dependence of $I_{0}$ on $a$. But first let us finish the proof of Proposition 4.1.

Turning now to the case $a<1$, we see from (4.20) that

$$
I_{\text {outer }} \approx-\frac{4 \Omega}{1-a^{2}} \int_{0}^{\frac{\pi}{2}}(\sin \theta)^{\frac{2}{a+1}} d \theta .
$$

Using (4.12) and (4.27),

$$
\begin{aligned}
I_{\text {inner }} & \approx-\frac{4}{1-a^{2}} \Omega^{\frac{a+2}{2 a+1}} \phi_{0}^{\frac{1-a}{a+1}} \\
& =-\frac{4}{1-a^{2}} \theta_{0}^{\frac{1-a}{a+1}} \Omega .
\end{aligned}
$$

Then $\left|I_{\text {outer }}\right| \gg\left|I_{\text {inner }}\right|$ since $\theta_{0} \ll 1$, and so

$$
A(\Omega) \approx \frac{4}{1-a^{2}} \Omega \int_{0}^{\frac{\pi}{2}}(\sin \theta)^{\frac{2}{a+1}} d \theta .
$$

Finally, consider the case $a=1$. We have

$$
\begin{aligned}
I_{\text {outer }} & \approx-\Omega \int_{\theta_{0}}^{\frac{\pi}{2}} \frac{(\cos \theta)^{2}}{\sin \theta} d \theta \\
& \approx \Omega \ln \theta_{0}
\end{aligned}
$$

since $\theta_{0} \ll 1$, and

$$
\begin{aligned}
I_{\text {inner }} & \approx 2 \Omega \int_{-\phi_{0}}^{\phi_{0}} y_{1}(\phi) d \phi \\
& \approx-\Omega \ln \phi_{0} .
\end{aligned}
$$

To derive (4.34), we used (4.12) with $a=1$ to approximate the integrand $y_{1}$. The first term in (4.12) integrates to zero because it is odd. Thus the dominant contribution to the integral comes from next term in (4.12), which decays like $1 /|\phi|$ for large $|\phi|$ and thereby yields the logarithmic dependence in (4.34). The asymptotic expansion for $y_{1}$ is not valid for small $|\phi|$, but that region makes only an $O(1)$ contribution to the integral and so is negligible in any case.

Next observe that $\theta_{0}=\Omega^{\frac{a+1}{2 a+1}} \phi_{0}=\Omega^{\frac{2}{3}} \phi_{0}$ from (4.19). So $A(\Omega)=\left|I_{\text {outer }}+I_{\text {inner }}\right|$ reduces to

$$
A(\Omega) \approx-\frac{2}{3} \Omega \ln \Omega
$$

and this completes the proof of the proposition.

4.2. Calculating the prefactor $\boldsymbol{I}_{\mathbf{0}}$. We have obtained explicit asymptotic formulas for $A(\Omega)$ when $a<1$ and $a=1$, but when $a>1$ the scaling law (4.29) depends on the integral $I_{0}$ defined in (4.25). We calculate $I_{0}$ next.

Using dominant balance in (4.11), we find that the asymptotic behavior of $y_{1}$ is

$$
y_{1} \approx-|\phi|^{\frac{1}{a+1}}-\frac{|\phi|^{\frac{-2 a}{a+1}}}{(a+1)^{2}}+\frac{5 a|\phi|^{\frac{-1-4 a}{a+1}}}{2(a+1)^{4}}-\frac{a(37 a+8)|\phi|^{\frac{-2-6 a}{a+1}}}{3(a+1)^{6}}
$$


as $\phi \rightarrow-\infty$ and

$$
y_{1} \approx \phi^{\frac{1}{a+1}}-\frac{\phi^{\frac{-2 a}{a+1}}}{(a+1)^{2}}-\frac{5 a \phi^{\frac{-1-4 a}{a+1}}}{2(a+1)^{4}}-\frac{a(37 a+8) \phi^{\frac{-2-6 a}{a+1}}}{3(a+1)^{6}}
$$

as $\phi \rightarrow+\infty$. We now choose some large positive number $R$. In calculating $I_{0}$ we approximate $y_{1}$ by (4.36) for $\phi<-R$ and by (4.37) for $\phi>R$. For $-R<\phi<R$ we find $y_{1}$ by numerically integrating (4.11) with initial condition

$$
y_{1}(-R)=-R^{\frac{1}{a+1}}-\frac{R^{\frac{-2 a}{a+1}}}{(a+1)^{2}}+\frac{5 a R^{\frac{-1-4 a}{a+1}}}{2(a+1)^{4}}-\frac{a(37 a+8) R^{\frac{-2-6 a}{a+1}}}{3(a+1)^{6}} .
$$

We get the following formula for $I_{0}$ :

$$
I_{0} \approx-\frac{4}{a^{2}-1} R^{\frac{1-a}{a+1}}-\frac{4 a(37 a+8)}{3(a+1)^{5}(1+5 a)} R^{\frac{-1-5 a}{a+1}}+2 \int_{-R}^{R} y_{1}(\phi) d \phi .
$$

The error we make is $O\left(R^{\frac{-2-7 a}{a+1}}\right)$. One can improve the accuracy by increasing $R$ or by calculating more terms in the expansion of $y_{1}$.

There are two nice limiting cases. From (4.39) we see that

$$
\left|I_{0}\right| \approx \frac{2}{a-1} \text { as } a \rightarrow 1^{+} .
$$

As $a \rightarrow+\infty,(4.11)$ goes to

$$
\frac{d y_{1}}{d \phi}= \begin{cases}+\infty & \text { if } y_{1}<-1, \\ \phi & \text { if }-1<y_{1}<1, \\ -\infty & \text { if } 1<y_{1},\end{cases}
$$

which can be solved explicitly to yield

$$
\left|I_{0}\right| \rightarrow \frac{16}{3} \text { as } a \rightarrow+\infty .
$$

To see this geometrically, Figure 4.1 shows the phase portrait of (4.11) for large $a$. The nullcline $d y_{1} / d \phi=0$ is given by the curve $y_{1}=f(\phi)$, which approaches a step function as $a \rightarrow+\infty$. The initial condition (4.38) starts below the nullcline. Then the flow rapidly brings the trajectory to the neighborhood of the nullcline branch $y_{1} \approx-1$. The solution drifts to the right until it is released at $\left(\phi, y_{1}\right) \approx(0,-1)$. Then integrating $d y_{1} / d \phi=\phi$ yields $y_{1}=-1+\frac{1}{2} \phi^{2}$, until $y_{1} \approx 1$ when $\phi \approx 2$. Thereafter $y_{1} \approx 1$ for $\phi \geq 2$. By substituting this $y_{1}(\phi)$ into $I_{0}$ and integrating, we obtain the desired result (4.42).

Figure 4.2 shows a plot of $\left|I_{0}\right|$ as a function of $a$ where we took $R=2$. (The approximation (4.39) works well even if $R$ is not very large, but from the case $a=+\infty$, we see that we need to take $R \geq 2$ at least.)

As a concrete example, our numerical calculations tell us that $\left|I_{0}\right|=81.1504$ when $a=1.025$, and the approximation $\left|I_{0}\right| \approx 2 /(a-1)$ implies $\left|I_{0}\right|=80$. For large $a$, say $a=40$, numerical calculations give $\left|I_{0}\right|=5.0416$ whereas (4.42) gives 5.333.

Tables 4.1 and 4.2 show some further numerical calculations to test the predicted exponents in the scaling law (4.29). The tables have the same format as Tables 3.1 and 3.2. For $F(x)=x^{3}$ and $F(x)=x^{5}$, the predicted exponents are $\beta=\frac{4}{5}$, and $\beta=\frac{2}{3}$, respectively. 


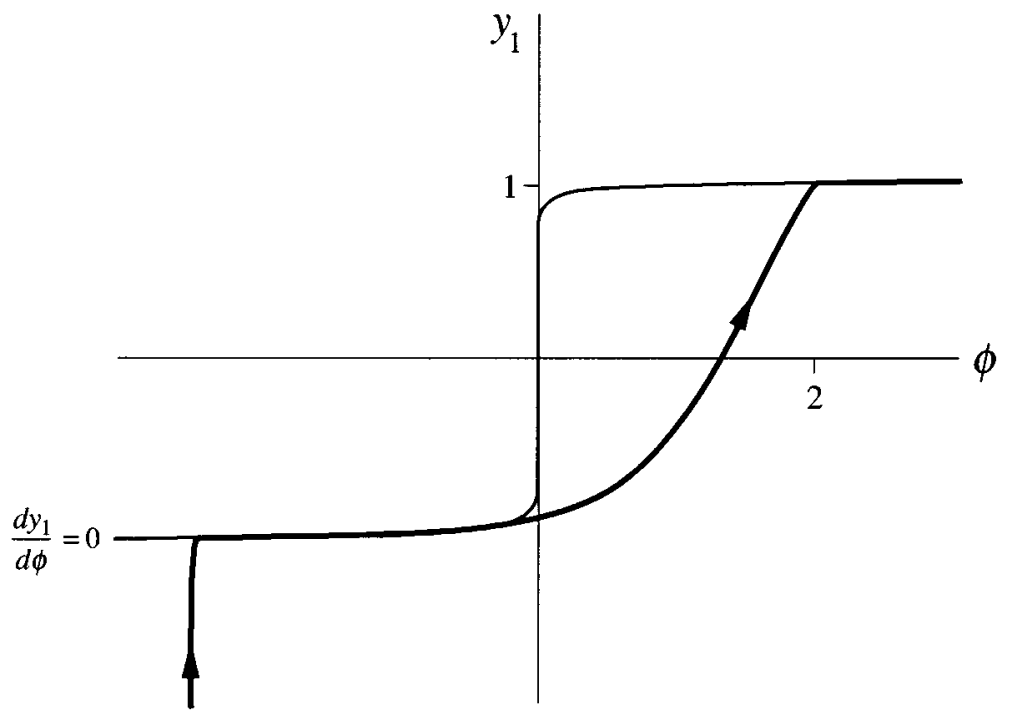

FIG. 4.1. Flow of (4.11) for large a.

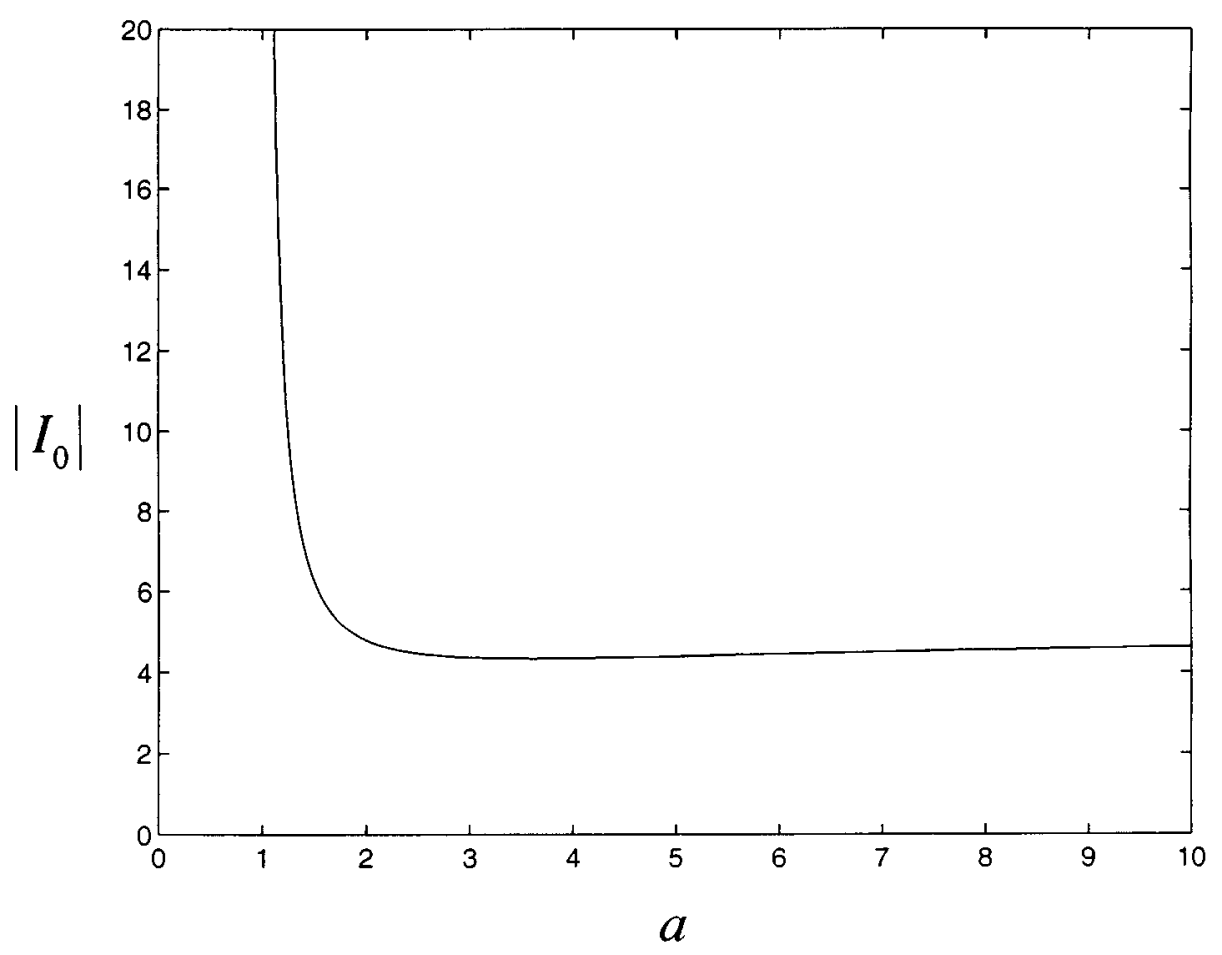

FIG. 4.2. Numerical evaluation of $\left|I_{0}\right|$ as a function of the exponent a.

Remark 2. We are now in a position to extend the result of Proposition 3.1 to the case where $F^{\prime}(x)=0$ for some $x$ such that $-E<F(x)<E$. Using the same procedure as in the proof of Proposition 4.1, we construct the outer approxi- 
TABLE 4.1

\begin{tabular}{|c|l|c|c|c|}
\hline \multicolumn{5}{|c|}{$F(x)=x^{3}$} \\
\hline$i$ & $\Omega_{i}$ & $A\left(\Omega_{i}\right)$ & $\beta_{i}$ & $A_{0}\left(\Omega_{i}\right)$ \\
\hline 1 & 0.1 & 0.6769 & 0.7442 & 0.7594 \\
2 & $0.1 \times 2^{-1}$ & 0.4041 & 0.7597 & 0.4361 \\
3 & $0.1 \times 2^{-2}$ & 0.2387 & 0.7684 & 0.2505 \\
4 & $0.1 \times 2^{-3}$ & 0.1401 & 0.7735 & 0.1439 \\
5 & $0.1 \times 2^{-4}$ & 0.0820 & 0.7794 & 0.0826 \\
6 & $0.1 \times 2^{-5}$ & 0.0478 & & 0.0475 \\
\hline \multicolumn{5}{|c|}{ Predicted exponent $\beta=0.8000$} \\
\hline
\end{tabular}

TABLE 4.2

\begin{tabular}{|c|l|l|l|l|}
\hline \multicolumn{5}{|c|}{$F(x)=x^{5}$} \\
\hline$i$ & $\Omega_{i}$ & $A\left(\Omega_{i}\right)$ & $\beta_{i}$ & $A_{0}\left(\Omega_{i}\right)$ \\
\hline 1 & 0.1 & 0.9108 & 0.6380 & 0.9379 \\
2 & $0.1 \times 2^{-1}$ & 0.5853 & 0.6502 & 0.5909 \\
3 & $0.1 \times 2^{-2}$ & 0.3729 & 0.6559 & 0.3722 \\
4 & $0.1 \times 2^{-3}$ & 0.2367 & 0.6599 & 0.2345 \\
5 & $0.1 \times 2^{-4}$ & 0.1498 & 0.6619 & 0.1477 \\
6 & $0.1 \times 2^{-5}$ & 0.0947 & & 0.0931 \\
\hline \multicolumn{5}{|c|}{ Predicted exponent $\beta=0.6666$} \\
\hline
\end{tabular}

mation to the periodic solution $z$. This outer approximation will fail for $\theta_{0}$ such that $F^{\prime}\left(x_{0}\left(\theta_{0}\right)\right)=0$ (see (3.9)). In this region we look at the behavior of $F(x)$. If $F(x)$ behaves like $F(x) \approx E \sin \theta_{0}+c\left(x-x_{0}\left(\theta_{0}\right)\right)\left|x-x_{0}\left(\theta_{0}\right)\right|^{a}$, we apply Proposition 4.1 with some constant that will multiply the final formula. We do this for every $\theta_{0}$ such that $F^{\prime}\left(x_{0}\left(\theta_{0}\right)\right)=0$ and then note which terms provide the major contribution to $A(\Omega)$.

5. Large $\Omega$. In this section we study the asymptotic behavior of $A(\Omega)$ as $\Omega \rightarrow$ $\infty$. Earlier results in this direction have been obtained by Rao, Krishnamurthy, and Pandit [18].

Proposition 5.1. Consider the system

$$
\dot{x}=-F(x)+E \sin \Omega t,
$$

where $E$ and $\Omega$ are positive constants and $F(x)$ is a continuously differentiable function that is strictly increasing in a neighborhood of $q$, where $F(q)=0$. Then for $\Omega \gg E$ the area of the dynamical hysteresis loop for system (5.1) is proportional to $\Omega^{-1}$.

Proof. As usual, let $\theta=\Omega t$. Equation (5.1) becomes

$$
\frac{d x}{d \theta}=\frac{-F(x)+E \sin \theta}{\Omega} .
$$

Let $z(\theta)$ denote the periodic solution in the neighborhood of $q$, and expand $z$ as

$$
z=z_{0}+z_{1}+z_{2}+\cdots
$$

with $z_{i+1} \ll z_{i}$.

At the first approximation we have $d z_{0} / d \theta=0$, so $z_{0}$ is a constant. At the next order,

$$
\frac{d z_{1}}{d \theta}=\frac{-F\left(z_{0}\right)+E \sin \theta}{\Omega}
$$


where we have approximated $F(z)$ by $F\left(z_{0}\right)$. Since $z$ is periodic in $\theta$, we need $F\left(z_{0}\right)=$ 0 and

$$
\frac{d z_{1}}{d \theta}=\frac{E}{\Omega} \sin \theta
$$

Then $z_{0}=q$ and

$$
z_{1}=-\frac{E}{\Omega} \cos \theta+C
$$

Going one more order in (5.2) we get

$$
\frac{d z_{2}}{d \theta}=-\frac{F\left(q+C-\frac{E}{\Omega} \cos \theta\right)}{\Omega} .
$$

By again requiring periodicity of $z_{2}$, we infer that

$$
\int_{0}^{2 \pi} F\left(q+C-\frac{E}{\Omega} \cos \theta\right) d \theta=0 .
$$

Since $F(q)=0, C$ exists for $\Omega$ large enough and $C \rightarrow 0$ as $\Omega \rightarrow \infty$.

We conclude that the limit cycle is approximated by

$$
z=q+C-\frac{E}{\Omega} \cos \theta
$$

and hence the area of the dynamical hysteresis loop is

$$
A(\Omega) \approx \frac{\pi E^{2}}{\Omega}
$$

which proves the proposition.

In the analysis above, we never used the assumption that $F$ is locally increasing. The point is that the periodic solution $z$ will be stable only if $F$ is locally increasing; this was shown in the lemma at the beginning of section 2 .

We now compare the predicted area (5.10) against numerical computations for two different functions $F(x)$, using $E=1$ in both cases (see Tables 5.1 and 5.2). As in earlier tables, $A$ is the area computed numerically, and $A_{0}$ is the analytical approximation.

It is interesting to see that for $F(x)=x^{3}, A(\Omega)$ starts behaving like $\Omega^{-1}$ sooner than it does for $F(x)=x$. The reason is simple: in these two cases, $q=C=0$ (see the proof of Proposition 5.1), and so from (5.7), the first term we are neglecting in the expansion of our periodic solution, namely $z_{2}$, is $O\left(\Omega^{-2}\right)$ for $F(x)=x$ and $O\left(\Omega^{-4}\right)$ for $F(x)=x^{3}$.

6. Generalizations. The results obtained in the previous sections have natural generalizations, some of which we now state without proof. These generalizations are motivated in part by scientific applications to a bistable semiconductor laser system [9] and to magnetic spin systems, as will be discussed in section 7. The first three generalizations deal with low-frequency driving and the final generalization deals with high-frequency driving. 
TABLE 5.1

\begin{tabular}{|c|l|l|l|l|}
\hline \multicolumn{5}{|c|}{$F(x)=x$} \\
\hline$i$ & $\Omega_{i}$ & $A\left(\Omega_{i}\right)$ & $\beta_{i}$ & $A_{0}\left(\Omega_{i}\right)$ \\
\hline 1 & 10 & 0.3110 & -0.9892 & 0.3142 \\
2 & $10 \times 2^{1}$ & 0.1567 & -0.9973 & 0.1571 \\
3 & $10 \times 2^{2}$ & 0.0785 & -0.9993 & 0.0785 \\
4 & $10 \times 2^{3}$ & 0.0393 & -0.9998 & 0.0393 \\
5 & $10 \times 2^{4}$ & 0.0196 & -1.0000 & 0.0196 \\
6 & $10 \times 2^{5}$ & 0.0098 & & 0.0098 \\
\hline
\end{tabular}

TABLE 5.2

\begin{tabular}{|l|l|l|l|l|}
\hline \multicolumn{5}{|c|}{$F(x)=x^{3}$} \\
\hline$i$ & $\Omega_{i}$ & $A\left(\Omega_{i}\right)$ & $\beta_{i}$ & $A_{0}\left(\Omega_{i}\right)$ \\
\hline 1 & 1 & 2.3916 & -0.6187 & 3.1416 \\
2 & $2^{1}$ & 1.5576 & -0.9865 & 1.5708 \\
3 & $2^{2}$ & 0.7861 & -0.9998 & 0.7854 \\
4 & $2^{3}$ & 0.3931 & -1.0000 & 0.3927 \\
5 & $2^{4}$ & 0.1965 & -1.0001 & 0.1963 \\
6 & $2^{5}$ & 0.0983 & & 0.0982 \\
\hline
\end{tabular}

6.1. One-dimensional systems: Generic case. This generalization of Proposition 3.1 concerns a broader class of one-dimensional systems than we have previously considered. The periodic forcing can be nonsinusoidal and it can also appear more implicitly than as an additive term in the differential equation. But, we retain the earlier assumptions that the forcing is low frequency and that the system has no static hysteresis.

Let $F(x, y)$ be a real-valued continuously differentiable function. Let $g(\theta)$ be a periodic real-valued function with period $T$, whose image is the interval $\left[y_{L}, y_{U}\right]$. Suppose that the equation

$$
F\left(x^{*}(y), y\right)=0
$$

implicitly defines a continuous function of $y$ that we call $x^{*}(y)$ for $y_{L} \leq y \leq y_{U}$. (Equation (6.1) may have several solutions but we restrict our attention to only one branch.) Let

$$
\Omega_{0} \equiv \min _{y_{L} \leq y \leq y_{U}} \frac{\partial F}{\partial x}\left(x^{*}(y), y\right) .
$$

If $\Omega_{0}>0$, then for $\Omega \ll \Omega_{0}$ the system

$$
\dot{x}=-F(x, g(\Omega t))
$$

has a dynamical hysteresis area proportional to $\Omega$ :

$$
A(\Omega) \approx \Omega\left|\int_{0}^{T} F_{y}\left(x^{*}(g(\theta)), g(\theta)\right)\left(\frac{g^{\prime}(\theta)}{F_{x}\left(x^{*}(g(\theta)), g(\theta)\right)}\right)^{2} d \theta\right|,
$$

where now the dynamical hysteresis area is defined as the area enclosed in the plane by the curve

$$
\mathcal{C}_{\Omega}:(g(\Omega t), z(t))
$$


for $0 \leq t \leq T$. Here $z(t)$ is the periodic solution of (6.3) that approaches $x^{*}(g(\Omega t))$ as $\Omega \rightarrow 0$.

For a further generalization, one can replace $\mathcal{C}_{\Omega}$ by a more general curve

$$
\mathcal{C}_{\Omega}^{*}:(h(g(\Omega t)), j(z(t))),
$$

where $h$ and $j$ are continuous real-valued functions. The scaling law $A(\Omega) \propto \Omega$ will still hold, except in some pathological cases, e.g., if $h$ or $j$ is a constant function. Also, the integral that multiplies $\Omega$ will be altered slightly to include the dependence on $h$ and $j$.

6.2. Multidimensional systems: Generic case. The previous result can be extended to the case where the system (6.3) is multidimensional; now $F$ and $x$ are $n$-dimensional vectors. We define

$$
\lambda_{y} \equiv \min \left\{\operatorname{Re}(\lambda): \lambda \text { is an eigenvalue of } J\left(x^{*}(y)\right)\right\},
$$

where $J\left(x^{*}(y)\right)$ is the Jacobian matrix of $F,\left(\partial F_{i} / \partial x_{j}\right)$ evaluated at $x^{*}(y)$. Let

$$
\Omega_{0} \equiv \min _{y_{L} \leq y \leq y_{U}} \lambda_{y}
$$

If $\Omega_{0}>0$, then for $\Omega \ll \Omega_{0}$ the system (6.3) has a dynamical hysteresis area proportional to $\Omega$, where the dynamical hysteresis area is the area enclosed by the curve (6.6).

6.3. Threshold case. We can also extend the result of Proposition 4.1 (or Remark 2). Suppose that there is a $y_{0}$ such that $\frac{\partial F}{\partial x}\left(x^{*}\left(y_{0}\right), y_{0}\right)=0$ and that $\frac{\partial F}{\partial x}\left(x^{*}(y), y\right)>0$ for $y \neq y_{0}$. If near $\left(x^{*}\left(y_{0}\right), y_{0}\right), F$ behaves like

$$
F(x, y) \approx c_{1}\left(x-x^{*}\left(y_{0}\right)\right)\left|x-x^{*}\left(y_{0}\right)\right|^{a}+c_{2}\left(y-y_{0}\right)
$$

with $c_{1}>0, c_{2}$ and $\frac{d g}{d y}\left(y_{0}\right)$ different from 0 , the result of Proposition 4.1 is still valid. Furthermore, as in Remark 2, we can have more than one $y_{0}$ where $\frac{\partial F}{\partial x}\left(x^{*}\left(y_{0}\right), y_{0}\right)=0$.

Remark 3. We can also extend the results of Proposition 4.1 to several dimensions if, for each $y$ such that $\lambda_{y}=0$ (see (6.7)), only one eigenvalue of $J\left(x^{*}(y)\right)$ has real part 0 , and consequently vanishes.

This is an important case because it arises naturally at zero-eigenvalue bifurcations. For instance, in section 7 we will encounter cases where $F$ depends on an extra parameter $\mu$, and the system

$$
\dot{x}=-F(x, g(\Omega t), \mu)
$$

has static hysteresis for $\mu>\mu_{0}$ but not for $\mu \leq \mu_{0}$. At $\mu=\mu_{0}$ we have a zeroeigenvalue bifurcation. If $F$ is differentiable many times (for example, if $F$ is analytic), we can use center manifold theory to obtain the reduced dynamics of (6.10) near $\left(x^{*}\left(y_{0}\right), y_{0}\right)$ for $\mu=\mu_{0}$. The dynamics will typically be governed by an equation of the form

$$
\dot{x}=-c_{1} x^{3}+c_{2} \Omega\left(t-t_{0}\right),
$$

where $y_{0}$ and $t_{0}$ are such that $\lambda_{y_{0}}=0$ and $y_{0}=g\left(\Omega t_{0}\right)$. Then, by extending Proposition 4.1 for the case of exponent $a=2$, we can show that the area grows like $A(\Omega) \propto \Omega^{\frac{4}{5}}$. 
6.4. High-frequency driving. We turn now to extensions for large $\Omega$. For one dimension the system (6.3) has a dynamical hysteresis loop whose area is proportional to $\Omega^{-1}$ as $\Omega \rightarrow \infty$ if

$$
\int_{0}^{T} \frac{\partial F}{\partial x}\left(z^{*}, g(\theta)\right) d \theta>0
$$

where $T$ is the period of $g$ and $z^{*}$ is a solution of

$$
\int_{0}^{T} F\left(z^{*}, g(\theta)\right) d \theta=0
$$

The condition (6.12) is not necessary but it is an easy condition to test and if it happens to be satisfied, we know that the periodic solution is linearly stable.

In the multidimensional case, condition (6.12) has to be replaced by the requirement that all the solutions of the variational equation

$$
\frac{d v}{d \theta}=-J\left(z^{*}, g(\theta)\right) v
$$

decay to 0 as $\theta \rightarrow \infty$. Equation (6.13) stays the same but becomes a vector equation.

7. Application to magnetic spin systems. In this section, we compare our results to the numerical observations of Luse and Zangwill [14]. For other works where our theory applies, see $[4,9]$.

Luse and Zangwill [14] investigated the area of the dynamical hysteresis loop for three related mean-field kinetic Ising models. The governing equations are

$$
\begin{gathered}
\dot{M}=\frac{1}{\tau}\left(-M+\tanh \left[\frac{n J M+H \sin \Omega t}{k T}\right]\right), \\
\dot{M}=\frac{1}{\tau}\left(-M \cosh \left[\frac{n J M+H \sin \Omega t}{k T}\right]+\sinh \left[\frac{n J M+H \sin \Omega t}{k T}\right]\right), \\
\dot{M}=\frac{1}{\tau}\left(B M-C M^{3}+H \sin \Omega t\right),
\end{gathered}
$$

where $M$ is the average magnetization of an Ising model with $n$ nearest neighbors and ferromagnetic exchange $J, k$ is the Boltzmann constant, $T$ is the temperature, $B$ and $C$ in (7.3) are constants with $B \propto \frac{n J}{k}-T$, and $H$ and $\Omega$ are the amplitude and frequency of the applied magnetic field. It is easy to check that all three systems have static hysteresis for $T<T_{c}$, where the critical temperature $T_{c}$ is given by

$$
T_{c}=\frac{n J}{k} .
$$

For low-frequency driving in the statically hysteretic regime, Luse and Zangwill [14] found that all three systems obey the same scaling law:

$$
A(\Omega)-A_{0} \sim H^{\frac{2}{3}} \Omega^{\frac{2}{3}}
$$

for small $\Omega$ and $T<T_{c}$, where $A_{0}$ is the area of the static hysteresis loop. But they were unable to determine the low-frequency behavior of $A(\Omega)$ for $T \geq T_{c}$, i.e., in the absence of static hysteresis. 
For high-frequency driving, the numerical results appeared to fit the empirical scaling law

$$
A(\Omega) \sim \frac{H^{\frac{2+5 \gamma}{3}}}{\Omega}
$$

independently of $T$, as long as $\Omega$ and $\frac{\Omega}{H^{\gamma}}$ are large. The quantity $\gamma$ in the exponent depends on the system:

$$
\gamma= \begin{cases}0.3 & \text { for }(7.1) \\ 1.1 & \text { for }(7.2) \\ 0.82 & \text { for }(7.3)\end{cases}
$$

In the following, we summarize the scaling laws predicted by our theory and outline the derivation of those formulas. Because the algebraic manipulations are similar to those shown earlier, we omit many of the intermediate steps.

7.1. Large $\Omega$. Let us focus first on the high-frequency limit $\Omega \tau \gg 1$. Equation (5.10) can be applied directly to (7.3), because the periodic forcing appears as a simple additive term in (7.3). After appropriate rescaling of parameters, we find that

$$
A(\Omega) \approx \frac{\pi H^{2}}{\tau \Omega}
$$

for $B, C$, and $H$ all much smaller than $\tau \Omega$, giving $\gamma=0.8$, close to the numerical result 0.82 in (7.7).

To analyze (7.1) and (7.2), we need to supplement the techniques of section 5 with those of section 6.4, because the periodic forcing now appears inside the argument of a transcendental function rather than as a simple additive term. We let $\theta=\Omega t$ and approximate the periodic solution $z$ by $z_{0}+z_{1}$, where $z_{0}$ is a constant and

$$
\frac{d z_{1}}{d \theta}=-\frac{F\left(z_{0}, g(\theta)\right)}{\tau \Omega}
$$

Here $g(\theta)=E \sin \theta$ with

$$
E=\frac{H}{k T}
$$

and

$$
F(x, y)= \begin{cases}x-\tanh (\mu x+y) & \text { for }(7.1) \\ x \cosh (\mu x+y)-\sinh (\mu x+y) & \text { for }(7.2)\end{cases}
$$

where

$$
\mu=\frac{n J}{k T}
$$

Because of periodicity of $z_{1}$ we have that $z_{0}$ is a solution of

$$
\int_{0}^{2 \pi} F\left(z_{0}, g(\theta)\right) d \theta=0 .
$$


The area will then be given by

$$
\begin{aligned}
A(\Omega) & \approx\left|\int_{0}^{2 \pi} z_{1}(\theta) g^{\prime}(\theta) d \theta\right| \\
& =\left|\int_{0}^{2 \pi} \frac{d z_{1}}{d \theta}(\theta) g(\theta) d \theta\right| \\
& =(\Omega \tau)^{-1}\left|\int_{0}^{2 \pi} F\left(z_{0}, g(\theta)\right) g(\theta) d \theta\right| .
\end{aligned}
$$

Some work is now required to approximate this integral. The calculations are done first for (7.1) and then for (7.2).

7.1.1. Large $\boldsymbol{\Omega}$ for the system (7.1). For system (7.1), the approximations (7.9), (7.13), and (7.14) are valid for any $E$ because $\tanh (x)$ is a bounded function.

There are two important subcases to consider: weak forcing $E \ll 1$ and strong forcing $E \gg 1$.

We begin with $E \ll 1$. Then $z_{0}=z^{*}+O\left(E^{2}\right)$, where

$$
z^{*}-\tanh \left(\mu z^{*}\right)=0 .
$$

Equation (7.15) has three solutions for $\mu>1$ (corresponding to the statically hysteretic case $\left.T<T_{c}\right)$ and one solution for $\mu \leq 1\left(T \geq T_{c}\right)$. We are interested in the stable periodic solutions. From (6.12), those solutions satisfy

$$
\begin{aligned}
\int_{0}^{2 \pi} \frac{\partial F}{\partial x}\left(z_{0}, g(\theta)\right) d \theta & \approx 2 \pi \frac{\partial F}{\partial x}\left(z^{*}, 0\right) \\
& =2 \pi-2 \pi \mu \cosh ^{-2}\left(\mu z^{*}\right) \\
& =-2 \pi \frac{\frac{\partial F}{\partial \mu}\left(z^{*}, 0\right)}{\frac{d z^{*}}{d \mu}} \\
& =2 \pi \frac{z^{*} \cosh ^{-2}\left(\mu z^{*}\right)}{\frac{d z^{*}}{d \mu}} \\
& >0
\end{aligned}
$$

Here we have expanded around $\left(z^{*}, 0\right)$ since $E$ is small and, therefore, $g(\theta)$ is small. Terms of order $E^{2}$ are neglected and we regard $z^{*}$ as a function of $\mu$ given implicitly by (7.15). We see that for $\mu \geq 1$ we have three different functions $z_{i}^{*}(\mu)$ with $z_{i}^{*}(1)=0$, $z_{1}^{*}(+\infty)=-1, z_{2}^{*}(\mu)=0$ for all $\mu, z_{1}^{*}$ is decreasing, and $z_{3}^{*}=-z_{1}^{*}$. From (7.16) and these observations about the behavior of the different $z_{i}^{*}(\mu)$ we infer that for $\mu>1$, $z_{1}^{*}(\mu)$ and $z_{3}^{*}(\mu)$ correspond to the stable periodic solutions and $z^{*}=0$ corresponds to the unstable one. For $\mu \leq 1$ there is only one periodic solution; it is stable and $z^{*}=0$. Expanding $F\left(z^{*}, y\right)$ around $y=0$ and substituting (7.14) we find that the area of the dynamical hysteresis loop is

$$
A(\Omega) \approx \frac{\pi E^{2}}{\Omega \tau} \cosh ^{-2}\left(\mu z^{*}\right)
$$

for system (7.1) in the limits $\Omega \tau \gg 1$ and $E \ll 1$.

Next we consider $E \gg 1$. Then

$$
\frac{\partial F}{\partial x}(0, g(\theta))=1-\mu \cosh ^{-2}(g(\theta))
$$


and so

$$
\int_{0}^{2 \pi} \frac{\partial F}{\partial x}(0, g(\theta)) d \theta=2 \pi+O\left(\frac{\mu}{E}\right)
$$

indicating that the periodic solution around 0 is stable. The three periodic solutions have collapsed to only one (because it is easy to see that any other periodic solution will be centered around a point near 0 and will be stable, but if we have more than one stable periodic solution we need an unstable one in between). Since

$$
\lim _{E \rightarrow+\infty} F(0, g(\theta))=\left\{\begin{aligned}
-1 & \text { if } \sin \theta>0 \\
1 & \text { if } \sin \theta<0
\end{aligned}\right.
$$

we get

$$
A(\Omega) \approx \frac{4 E}{\Omega \tau} .
$$

Comparing our analytical results to the numerical scaling law (7.6), we see that there is no uniform $\gamma$ for the system (7.1) independent of $T$. We find $\gamma=0.8$ for $H \ll k T$ (see (7.17)) whereas $\gamma=0.2$ for $H \gg k T$ (see (7.21)). It is interesting that Luse and Zangwill [14] found a value of $\gamma=0.3$ in between the two limiting values.

7.1.2. Large $\boldsymbol{\Omega}$ for the system (7.2). Now we repeat the calculations above but for system (7.2). The approximations (7.9), (7.13), and (7.14) are found to be valid only for $\Omega \tau \gg e^{E}$. In this regime, (7.13) implies that

$$
z_{0}-\tanh \left(\mu z_{0}\right)=0 \text {. }
$$

For $E \ll 1$, arguments similar to those used earlier show that

$$
A(\Omega) \approx \frac{\pi E^{2}}{\Omega \tau}\left(\cosh \left(\mu z_{0}\right)-z_{0} \sinh \left(\mu z_{0}\right)\right) .
$$

However, for $\Omega \tau \ll e^{E}$ the situation is different. In this case (7.9) is not valid. If $x<-1, F(x, y)<0$ and if $x>1, F(x, y)>0$. As a consequence the periodic solution $z(\theta)$ (and there is only one) satisfies $-1<z<1$. Dominant balance shows that

$$
z=\left\{\begin{aligned}
1 & \text { if } 0<\theta<\pi, E \theta \gg 1 \text { and } E(\pi-\theta) \gg 1 \\
-1 & \text { if }-\pi<\theta<0,|E \theta| \gg 1 \text { and }|E(\pi+\theta)| \gg 1
\end{aligned}\right.
$$

where we have neglected terms of order $e^{-E}$.

The asymptotic behavior of $z$ when (7.24) is not valid is given by $x(E \theta)$ for $\theta \ll 1$ and $-x(E(\theta-\pi))$ for $\theta-\pi \ll 1$, where $x$ satisfies

$$
\frac{d x}{d \phi}=\frac{-x+1}{2 \tau \Omega E} e^{\mu x+\phi}-\frac{x+1}{2 \tau \Omega E} e^{-\mu x-\phi}
$$

with boundary conditions $x \rightarrow-1$ as $\phi \rightarrow-\infty$.

Then the leading order approximation to the area is

$$
A(\Omega) \approx 2 \lim _{\phi_{0} \rightarrow \infty} \int_{-\phi_{0}}^{+\phi_{0}} x(\phi) d \phi=O(\ln (2 \tau \Omega E)) .
$$

To derive this result, we argue that (7.25) shows that $x$ is not close to 1 or -1 for a distance of order $\ln (2 \tau \Omega E)$; then we use similar techniques to those used in the proof of Proposition 4.1.

We see that the dependence on $H$ implied by (7.6) is incorrect. 
7.2. Small $\Omega$. We now analyze the models in the low-frequency limit $\Omega \tau \ll 1$.

Assume first that $T<T_{c}$, so that all three systems have static hysteresis. Jung, Gray, Roy, and Mandel [11] showed analytically that the scaling law (7.5) is correct for system (7.3). Those authors also argued that the scaling law should hold more generally, consistent with the numerical results of Luse and Zangwill [14] for systems (7.1) and (7.2).

For the threshold case $\mu=1$ (i.e., $T=T_{c}$ ), we can rescale the systems so that Proposition 4.1 applies. We find that when $\tau \Omega \ll E$, the area of the dynamical hysteresis loop for both systems (7.1) and (7.2) is

$$
A(\Omega) \approx\left|I_{0}\right|(27)^{\frac{1}{5}}(\tau \Omega E)^{\frac{4}{5}},
$$

where $I_{0}$ is defined in (4.25) and computed in section 4.2. The area for system (7.3) with $\tau \Omega \ll H$ and $\tau \Omega \ll C$ is given by

$$
A(\Omega) \approx\left|I_{0}\right| C^{-\frac{3}{5}}(\tau \Omega H)^{\frac{4}{5}} .
$$

Finally, when $\mu<1$ (i.e., $T>T_{c}$ ) we are in a position to apply the generalization discussed in section 6.1. For all three systems, the area of the hysteresis loop is proportional to $\Omega$ in the low-frequency limit. Specifically, if $\tau \Omega \ll E$ (and also $\tau \Omega \ll|B|$ for (7.3)), the area of the dynamical hysteresis loop is

$$
A(\Omega) \approx I \Omega \tau,
$$

where the prefactor $I$ depends in a complicated way on the system and the parameters $H$ and $E$, as follows. For the system (7.1),

$$
I=I_{1}=E^{2} \int_{0}^{2 \pi} \frac{\cosh ^{2}\left(\mu z_{1}(\theta)+E \sin \theta\right)}{\left(\cosh ^{2}\left(\mu z_{1}(\theta)+E \sin \theta\right)-\mu\right)^{2}} \cos ^{2} \theta d \theta .
$$

For (7.2),

$$
I=I_{2}=E^{2} \int_{0}^{2 \pi} \frac{1-z_{2}(\theta) \tanh \left(\mu z_{2}(\theta)+E \sin \theta\right)}{\left(1-\mu+\mu z_{2}(\theta) \tanh \left(\mu z_{2}(\theta)+E \sin \theta\right)\right)^{2}} \frac{\cos ^{2} \theta}{\cosh \left(\mu z_{2}(\theta)+E \sin \theta\right)} d \theta .
$$

Last, for (7.3),

$$
I=I_{3}=H^{2} \int_{0}^{2 \pi} \frac{\cos ^{2} \theta}{\left(-B+3 C\left(z_{3}(\theta)\right)^{2}\right)^{2}} d \theta .
$$

In the integrals above, $z_{1}$ and $z_{2}$ are the same periodic function, defined implicitly by $z(\theta)-\tanh (\mu z(\theta)+E \sin \theta)=0$. The function $z_{3}$ satisfies $B z_{3}-C\left(z_{3}\right)^{3}+H \sin \theta=0$.

To clarify the dependence on $E$ in the integrals $I_{1}$ and $I_{2}$, let us consider two limiting cases. When $E \ll 1, I_{1} \approx I_{2} \approx \pi E^{2} /(1-\mu)^{2}$. However, for $E \gg 1$,

$$
I_{1} \approx 2 E \int_{-\infty}^{+\infty} \frac{\cosh ^{2}(\mu y(\phi)+\phi)}{\left(\cosh ^{2}(\mu y(\phi)+\phi)-\mu\right)^{2}} d \phi
$$

where $y(\phi)-\tanh (\mu y(\phi)+\phi)=0$, and

$$
I_{2} \approx 2 E \int_{-\infty}^{+\infty} \frac{1-y(\phi) \tanh (\mu y(\phi)+\phi)}{(1-\mu+\mu y(\phi) \tanh (\mu y(\phi)+\phi))^{2}} \cosh ^{-1}(\mu y(\phi)+\phi) d \phi
$$


Thus we find that for both (7.1) and (7.2) the area goes like $A \sim E^{2} \Omega \tau$ for small $E$ and like $A \sim E \Omega \tau$ for large $E$.

Turning now to the integral $I_{3}$, we find that $I_{3} \approx \pi H^{2} / B^{2}$ when $H \ll 1$, whereas for $H \gg \max \{1, C,|B|\}$,

$$
I_{3} \approx 2 H \int_{-\infty}^{+\infty} \frac{d \phi}{\left(B-3 C\left(y_{3}(\phi)\right)^{2}\right)^{2}} d \theta,
$$

where $B y_{3}(\phi)-C\left(y_{3}(\phi)\right)^{3}+\phi=0$. Hence the area for (7.3) scales as $A \sim H^{2} \Omega \tau$ for small $H$ and $A \sim H \Omega \tau$ for large $H$.

Acknowledgments. We thank Raj Roy for helpful suggestions and for introducing us to the subject of dynamical hysteresis. Thanks to Andrew Zangwill for his comments on a draft of this paper.

\section{REFERENCES}

[1] M. Acharyya, B.K. Chakrabati, And A. Sen, Monte Carlo study of hysteretic response for the two dimensional Ising system: Scaling behavior, Phys. A, 186 (1992), pp. 231-236.

[2] M. Acharyya And B.K. Chakrabati, Monte Carlo study of hysteretic response and relaxation in Ising models, Phys. A, 192 (1993), pp. 471-485.

[3] M. Acharyya And B.K. Chakrabati, Magnetic hysteresis loops as Lissajous plots of relaxationally delayed response to periodic field variation, Phys. A, 202 (1994), pp. 467-481.

[4] M. Acharyya, B.K. Chakrabati, And R.B. Stinchcombe, Hysteresis in Ising model in transverse field, J. Phys. A: Math. Gen., 27 (1994), pp. 1533-1540.

[5] G. Bertotti, Generalized Preisach model for the description of hysteresis and eddy current effects in metallic ferromagnetic material, J. Appl. Phys., 69 (1991), pp. 4608-4610.

[6] G. Bertotti, Dynamic generalization of the scalar Preisach model of hysteresis, IEEE Trans. on Magnetics, 28 (1992), pp. 2599-2601.

[7] D. Dhar and P. Thomas, Hysteresis and self-organized criticality in the $O(N)$ model in the limit $N \rightarrow \infty$, J. Phys. A: Math. Gen. 25 (1992), pp. 4967-4984.

[8] Y.L. HE AND G.C. WANG, Observation of dynamic scaling of magnetic hysteresis in ultrathin ferromagnetic Fe/Au(001) films, Phys. Rev. Lett., 70 (1993), pp. 2336-2339.

[9] A. Hohl, H.J.C. van der Linden, R. Roy, G. Goldsztein, F. Broner, and S.H. Strogatz, Scaling laws for dynamical hysteresis in a multidimensional laser system, Phys. Rev. Lett., 74 (1995), pp. 2220-2223.

[10] D.C. JiLES, Frequency dependence of hysteresis curves in conducting magnetic materials, J. Appl. Phys., 76 (1994), pp. 5849-5855.

[11] P. Jung, G. Gray, R. Roy, And P. Mandel, Scaling law for dynamical hysteresis, Phys. Rev. Lett., 65 (1990), pp. 1873-1876.

[12] I. Lin And J. Lui, Dynamical double hysteresis in weakly ionized magnetoplasmas, Phys. Rev. A, 46 (1992), pp. R733-R736.

[13] W.S. Lo AND R.A. Pelcovits, Ising model in a time-dependent magnetic field, Phys. Rev. A, 42 (1990), pp. $7471-7474$.

[14] C.N. Luse and A. Zangwill, Discontinuous scaling of hysteresis losses, Phys. Rev. E, 50 (1994), pp. 224-226.

[15] M.C. Mahato And S.R. Shenoy, Hysteresis as a rate competition: A Landau model example, Phys. A, 186 (1992), pp. 220-230.

[16] M.C. Mahato AND S.R. Shenoy, Langevin dynamic simulation of hysteresis in a field-swept Landau potential, J. Statist. Phys., 73 (1993), pp. 123-145.

[17] M.C. Mahato And S.R. Shenoy, Hysteresis loss and stochastic resonance: A numerical study of a double-well potential, Phys. Rev. E, 50 (1994), pp. 2503-2512.

[18] M. Rao, H.R. Krishnamurthy, and R. Pandit, Magnetic hysteresis in two model spin systems, Phys. Rev. B, 42 (1990), pp. 856-884.

[19] M. RAO AND R. PANDit, Magnetic and thermal hysteresis in the $O(N)$-symmetric $\left(\Phi^{2}\right)^{3}$ model, Phys. Rev. B, 43 (1991), pp. 3373-3386.

[20] S. Sengupta, Y. Marathe, And S. Puri, Cell-dynamic simulation of magnetic hysteresis in two-dimensional Ising systems, Phys. Rev. B, 45 (1992), pp. 7828-7831.

[21] A. Somosa And R. Desai, Kinetics of systems with continuous symmetry under the effect of an external field, Phys. Rev. Lett., 70 (1993), pp. 3279-3282. 
[22] P. Thomas And D. Dhar, Hysteresis in isotropic spin systems, J. Phys A: Math. Gen., 26 (1993), pp. 3973-3981.

[23] M. .C. Torrent And M. San Miguel, Stochastic-dynamics characterization of delayed laser threshold instability with swept control parameter, Phys. Rev. A, 38 (1988), pp. 245-251.

[24] H. Zhong, J.X. ZhAng, And G.G. SiU, Dynamic scaling of hysteresis in a linearly driven system, J. Phys.: Condensed Matter, 6 (1994), pp. 7785-7796. 